This is an electronic reprint of the original article. This reprint may differ from the original in pagination and typographic detail.

Author(s): Niemelä, Marko; Kulmala, Juha-Pekka; Kauppi, Jukka-Pekka; Kosonen, Jukka; Äyrämö, Sami

Title: $\quad$ Prediction of active peak force using a multilayer perceptron

Year: $\quad 2017$

Version:

Please cite the original version:

Niemelä, M., Kulmala, J.-P., Kauppi, J.-P., Kosonen, J., \& Äyrämö, S. (2017). Prediction of active peak force using a multilayer perceptron. Sports Engineering, 20(3), 213219. https://doi.org/10.1007/s12283-017-0236-z

All material supplied via JYX is protected by copyright and other intellectual property rights, and duplication or sale of all or part of any of the repository collections is not permitted, except that material may be duplicated by you for your research use or educational purposes in electronic or print form. You must obtain permission for any other use. Electronic or print copies may not be offered, whether for sale or otherwise to anyone who is not an authorised user. 


\title{
Prediction of active peak force using a multilayer perceptron
}

\author{
Marko Niemelä ${ }^{1,2,3} \cdot$ Juha-Pekka Kulmala $^{1,4} \cdot$ Jukka-Pekka Kauppi $^{3,5} \cdot$ Jukka $^{2}$ \\ Kosonen $^{1,6}$. Sami Äyrämö $\ddot{1}^{1,3}$
}

Received: date / Accepted: date

\begin{abstract}
Both kinematic parameters and ground reaction forces are necessary for understanding the biomechanics of running. Kinematic information of a runner is typically measured by a motion capture system whereas ground reaction force during the support phase of running is measured by force platforms. In order to analyze both kinematics and kinetics of a runner over several subsequent contacts, an instrumented treadmill or alternatively several force platforms installed over a regulated space are available options, but they are highly immovable, expensive, and sometimes even impractical options. Naturally, it would be highly useful to predict ground reaction forces using a motion capture system only and this way reduce costs and complexity of the analysis. In this study, the machine learning model for vertical ground reaction force magnitude prediction based on running motion information of 128 healthy adults is pro-
\end{abstract}

\section{Marko Niemelä}

Niilo Mäki Institute, P.O.Box 35

FIN-40014 University of Jyväskylä

FINLAND

E-mail: marko.p.niemela@jyu.fi

${ }^{1}$ Agora Center, University of Jyväskylä, Jyväskylä, Finland.

${ }^{2}$ Niilo Mäki Institute, Jyväskylä, Finland.

3 Faculty of Information Technology, University of Jyväskylä, Jyväskylä, Finland.

4 Motion Analysis Laboratory, Children's Hospital, University of Helsinki and Helsinki University Hospital, Helsinki, Finland

${ }^{5}$ Department of Computer Science and HIIT, University of Helsinki, Helsinki, Finland

${ }^{6}$ Faculty of Sport and Health Sciences, University of Jyväskylä, Jyväskylä, Finland. posed. The predicted outputs of a multilayer perceptron model were compared with the actual force platform measurements. The results were evaluated with Pearson's correlation coefficient through a ten-fold cross validation. The mean standard error of the estimate was 0.107 body weights, showing that our method is sufficiently accurate to identify abnormalities in running technique among recreational runners.

Keywords Ground reaction force - Multilayer perceptron · Gait analysis · Running - Motion capture system · Force platform

\section{Introduction}

Lower extremity overuse injuries are very common in sports like running [1], where musculoskeletal structures are exposed to repeated loading cycles. The magnitude of force exerted on the ground during the stance phase of running provides information of the total leg force production as well as reflects the loads experienced by the musculoskeletal system. Therefore, ground reaction force (GRF) measurements have been typically used to evaluate the total mechanical loading that the lower extremities are exposed to during running [2].

Biomechanical running analysis typically requires a laboratory environment, where the GRFs and motion data are acquired simultaneously using a force platform and motion capture systems. As a result, such an analysis is seldom, if ever, available for measurements outside laboratory environment limiting its usability for technique monitoring and injury prevention among runners. While a collection of motion data is relatively easy to perform outside laboratory environment, force platforms are usually located in a regulated space and are not portable [3][4]. Moreover, restricted laboratory space may often be too strict for allowing runners to achieve constant speed and natural stepping on the force 
platform, particularly if only one force platform is used [4]. Given these issues and the fact that new markerless technologies [5][6] are making usability of motion capture systems even more flexible in the near future, there is need for developing new solutions for acquiring GRF information during human movement. Because motion capture systems are an integral part of gait analysis, the possibility to predict GRFs with reasonable accuracy solely based on motion data would be a great benefit, making it possible to perform gait analysis without expensive force platforms.

Previously, few studies have been conducted in an attempt to overcome the above mentioned limitations of the force platforms. For instance, in [7] an inverse dynamics method was utilized to calculate intersegmental forces and moments based on pure positional data of segment centers during gait. The study showed how the human body could be modeled as a combination of observed rigid segments. Another potential approach is to train a multivariate model on the basis of kinematic data to make predictions about physical quantities of interests. For instance, neural networks have been previously used to predict GRFs and moments from kinematic data during walking [3][8][4]. In [3], walking motions, trajectories, velocities, and accelerations of segment centers extracted from raw motion data were used as initial input variables into the neural network. Different data reduction techniques were used to limit input dimensionality. The predicted GRFs and moments were the model outputs. In [8], a three-layer neural network with wavelet activation functions in hidden layers was used. Thus, both the general approximation of neural networks and the localization property of wavelets were utilized for the model design. The model was reported to be stable, robust, high level of accuracy and fast [8]. In [9], force magnitude, stiffness, and elastic energy based on full body kinematics during running were estimated. Filtered and differentiated sagittal plane center of mass information was used to obtain accelerations. After taking into account body mass, the analogous force was estimated and the results were compared against the GRF data. The study showed that the force magnitude, stiffness, and elastic energy could be estimated precisely. However, the results depended on filter settings that were applied to kinematics [9].

While neural networks have been successively used in different areas of sport, running prediction based on running kinematics have not been reported. In this work, we used a multilayer neural network model to predict peak vertical GRFs. We calculated input parameters of the network model using body center of mass kinematics and foot markers trajectories during the stance phase of running. Force platform measurements were used to train the network to predict a vertical peak value of the GRF.

Detailed assessment of technique and total limb loading in running includes several variables of interest. Among

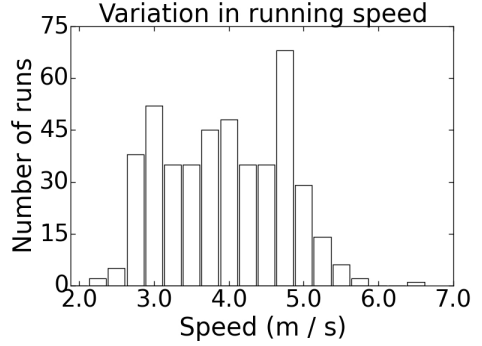

Fig. 1: Running speed histogram of 128 participants

these variables, the peak force is of primary interest since it reflects the peak loading exposed to lower extremities over the period of ground contact. Thus, the choice of the peak force as an outcome variable in the MLP provides a good basis for the study.

\section{Methods}

\subsection{Experimental protocol}

The study was approved by the local ethics committee, which was performed in accordance with the Declaration of Helsinki. The 3D motion analysis running an indoor sports hall volunteered in total 128 healthy participants, including 82 males and 46 females (height: $174.9 \pm 9.2 \mathrm{~cm}$; mass: $71.4 \pm 11.4$ $\mathrm{kg}$; body mass index: $23.2 \pm 2.3 \mathrm{~kg} / \mathrm{m}^{2}$ ). The participants were questioned before the runs to confirm that no clinical problems affected to the running pattern. The participants were advised to use their own shoes and run at predefined running speed. All the participants performed in total 450 runs at different speeds as shown in the Figure 1. One to six runs per participant were selected for data processing and neural network training, as each run operated as one input sample for a network model.

\subsection{Instrumentation}

Retroreflective markers were placed on the participant's body according to the Vicon plug-in gait lower body model (16 markers in total). The 3D motion of the markers was recorded at a sampling frequency of $300 \mathrm{~Hz}$ using a motion analysis system of eight infrared cameras (Vicon T40, Vicon, Oxford, UK). The participants performed several running trials over a force platform (AMTI BP6001200, AMTI, Watertown, MA, USA) which was mounted in the middle of a 50 meter runway. Ground reaction data was recorded synchronously with kinematic data at a sampling frequency of $1500 \mathrm{~Hz}$. For each participant a static calibration trial was used to define the anatomical coordinate system. The global $\mathrm{X}$-axis was set in the direction of progression, the $\mathrm{Y}$-axis 
was medio-lateral, and the Z-axis was vertical. Vicon Nexus (v. 1.7.1) software was used to collect kinematic and GRF data in C3D file format. The participant's running speed was monitored with two photocells placed on each side of the force platform and $6 \mathrm{~m}$ apart.

\subsection{Data processing}

Both the kinematic and GRF data were filtered with a fourthorder Butterworth, zero-lag, low pass digital filter. The cutoff frequency was $12 \mathrm{~Hz}$ for the kinematic data and $50 \mathrm{~Hz}$ for the GRF data. The standard Vicon plug-in gait model was used to calculate joint kinematics in each trial. The single value of each of following measures was calculated from the first contact cycle: speed, contact time, center of mass location, overstriding, stride length, vertical oscillation, vertical velocity, and vertical acceleration. Speed was obtained from the photocells and contact time was estimated from the positions of the heel and toe markers (see section 2.5 for details). The location of the participant's center of mass was taken as the arithmetic mean of the four pelvic markers (left anterior superior iliac spine, right anterior superior iliac spine, left posterior superior iliac spine, right posterior superior iliac spine). Overstriding was taken as the horizontal distance between the center of mass and heel marker at the instant of the initial ground contact. Stride length was taken as the horizontal distance between the left and right leg ankle markers at two consecutive ground contacts. Pelvis vertical oscillation was taken as the vertical distance between the highest and lowest points of the body center of mass during the stride. Vertical velocity and vertical acceleration were taken as the maximum values of the center of mass velocity and acceleration during the period of ground contact.

Initial ground contact and toe-off were captured from heel and toe marker positions in running. The marker positions were compared to reference values which were recorded during the static calibration session. Two offset terms were selected based on the running data of all the participants to obtain better accuracies for estimated initial ground contact and toe-off times. The offset terms were added to the reference values. The offset term was $-1 \mathrm{~mm}$ for the initial contact and $4 \mathrm{~mm}$ for the toe-off. Estimated contact time was the time between the initial ground contact and toe-off. Estimated times were compared against real contact times extracted from the GRF data. Example trajectories of heel and toe markers are shown in Figure 2.

\subsection{Background of neural networks}

Neural networks are statistical learning models attempting to simulate biological neural systems [10]. Neural networks are of great interest in many fields of science because of
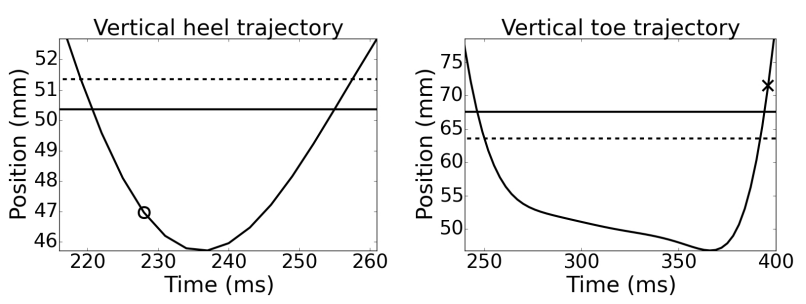

Fig. 2: Vertical heel and toe marker trajectories. The reference positions of markers are illustrated as dotted lines. The offset positions are marked as solid lines. The real initial contact (circle) and toe-off (cross) were measured from a force platform

their ability to accurately approximate complex non-linear functions of their inputs and are thus well-suited for many real-world data analysis tasks. A neural network calculates parameters based on training data and is useful in situations where there is not much prior information for task completion. One of the simplest model of a neural network is called the perceptron [11]. The perceptron produces its output value by computing a weighted sum of its inputs, subtracting a bias term from the sum, and then examining the sign of the result. The perceptron acts as a binary classifier, where the output (either -1 or +1 ) determines the category of the input data point.

Multilayer perceptron (MLP) is an extension of the perceptron for multiple feed-forward-way interconnected layers of nodes and non-linear activation functions [12]. The first layer that interacts with the environment is known as the input layer. The final layer that presents the processed data is the output layer. Intermediate levels between input and output layers are called hidden layers. Except for the input layer units, each node of the MLP is called a neuron, which contain an activation function. The activation functions not need to be binary valued and therefore MLP network cannot only be used for classification but for non-linear regression of continuous-valued outputs.

MLPs with at least one hidden layer are universal approximators [13], meaning that they can approximate target functions of arbitrary complexity. The downside of neural networks is overfitting, which occur when an overly complex neural network model describes random error or noise instead of the underlying relationship. To avoid overfitting, it is important to choose an appropriate network complexity, topology and cost function for a given problem.

\subsection{MLP Cost function}

Before predictions can be made using the MLP, a learning algorithm must be run to determine a set of weights that minimize the value of the cost function, which is usually 
chosen to be the sum of squares of the network errors on the training data set. The mean square error is calculated as

$F=\frac{1}{N} \sum_{i=1}^{N}\left(y_{i}-\hat{y}_{i}\right)^{2}$

where $N$ is the number of training samples, $y_{i}$ is the target value and $\hat{y}_{i}$ is the predicted value. It is possible to improve generalization by modifying the cost function by adding a penalty term $\mathbf{w}$ that consists of the network weights and biases [14]. The penalized mean square error is calculated as

$F=\alpha \frac{1}{N} \sum_{i=1}^{N}\left(y_{i}-\hat{y}_{i}\right)^{2}+\beta \frac{1}{K} \sum_{j=1}^{K} w_{j}^{2}$

where $K$ is the total number of weights and biases. The regularization parameters $\alpha$ and $\beta$ can be obtained by maximizing a Bayesian posterior function during the network training [14]. If a uniform density for the regularization parameters is assumed, the maximum posterior is achieved by means of maximum likelihood estimation. Using this penalized form causes the weights and biases to converge to smaller absolute values than they otherwise would. This forces the network response to be smoother and the model is less prone to overfitting.

\subsection{Feature extraction and MLP training}

An MLP architecture was used in this study and it is shown in Figure 3. MLP was used to approximate the nonlinear relationship between inputs and continuous-valued peak GRF outputs (i.e. non-linear regression was performed). The network was created using a Neural Network Toolbox (v. 8.4) of MATLAB (v. R2015b, 64-bit, The MathWorks, Inc). The inputs to the network were selected based on prior knowledge about the biomechanics of human motion. The selected features were weight (kilograms), speed (meters / second), contact time (seconds), overstriding (meters), stride length (meters), vertical oscillation (meters), pelvis maximum vertical velocity (meters / second), and pelvis maximum vertical acceleration (meters / second ${ }^{2}$ ). The network output was the predicted peak GRF (body weights). The target force values needed in the model training and testing were obtained using force platforms.

The network inputs were linearly scaled between the range $[-1+1]$. A purelin transfer function was used in the output layer and a tan-sigmoid transfer function in the hidden layer. The hidden layer size was selected as six neurons on an empirical method basis. A Bayesian regularization training method was used in the experiments. The Bayesian regularization updated the weight and bias values according to the Jacobian backpropagation algorithm [14]. The method minimized a combination of squared error and weights, and produced the network that generalized well to unseen data.

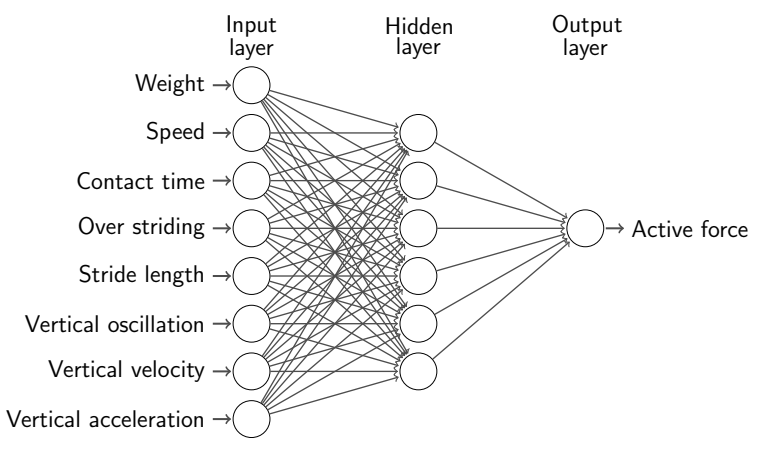

Fig. 3: An MLP architecture used in this study for prediction of active peak force value

The MATLAB Neural Network Toolbox's default training parameters were used as input parameters to the neural network model. The parameters were the maximum number of epochs to train (1000), the maximum value of the Marquardt adjustment parameter $(1.0 \mathrm{e}+10)$, and the minimum performance gradient (1.0 e-07). Training was automatically stopped when any of these conditions occurred. Network training was performed 100 times from random initial conditions of the network weights to avoid local minima of the error surface.

\subsection{Performance evaluation}

The performance was evaluated through a ten-fold cross validation technique. More specifically, the data was randomly divided into a test and training fold ten times such that the amount of training data was $90 \%$ in each fold and every sample was selected to test fold one time in total. The calculated active peak forces were compared with force platform data and similarities between the predicted and measured values were determined by calculating Pearson's correlation coefficient

$r=\frac{1}{N-1} \sum\left(\frac{y_{i}-\mu_{y}}{\sigma_{y}}\right)\left(\frac{\hat{y}_{i}-\mu_{\hat{y}}}{\sigma_{\hat{y}}}\right)$

and standard error of the estimate

$S_{\text {est }}=\sigma_{\hat{y}} \sqrt{1-r^{2}}$

In the above equations $N$ is the number of samples in a test data set, $\mu_{y}$ is the mean value of measured peak force values, $\mu_{\hat{y}}$ is the mean value of predicted peak force values, $\sigma_{y}$ is the standard deviation of measured values of active peak forces and $\sigma_{\hat{y}}$ is the standard deviation of predicted values of active peak forces. Finally, the arithmetic means of Pearson's correlation coefficient and standard error of the estimate values were calculated over ten test folds to form the final evaluation results. 
Table 1: Correlation coefficients and standard error of the estimates for active peak forces evaluated using a ten-fold cross validation

\begin{tabular}{lllll}
\hline & \multicolumn{2}{l}{$\begin{array}{l}\text { Pearson's correlation } \\
\text { coefficient }\end{array}$} & \multicolumn{2}{l}{$\begin{array}{l}\text { Standard error } \\
\text { of the estimate } \\
\text { (body weights) }\end{array}$} \\
\hline Folds 1 \& 2 & 0.915 & 0.947 & 0.109 & 0.087 \\
Folds 3 \& 4 & 0.882 & 0.924 & 0.132 & 0.100 \\
Folds 5 \& 6 & 0.951 & 0.908 & 0.075 & 0.116 \\
Folds 7 \& 8 & 0.929 & 0.901 & 0.103 & 0.116 \\
Folds 9 \& 10 & 0.916 & 0.912 & 0.119 & 0.115 \\
Mean & $\mathbf{0 . 9 1 9}$ & & $\mathbf{0 . 1 0 7}$ & \\
\hline
\end{tabular}

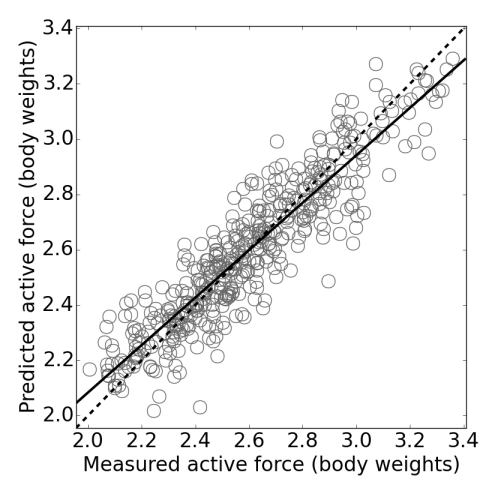

Fig. 4: Measured versus predicted active peak force based on multilayer perceptron. The regression line is marked as solid line and the line of identity is marked as dotted line

\section{Results}

According to Table 1, the average Pearson's correlation coefficient between the measured and predicted forces was 0.919 . The average standard error of the estimate was 0.107 body weights. Figure 4 shows the combined values, which were obtained when all the active peak force predictions were taken into account in the calculations. Clearly, the predicted forces fit very well to the measured values $(r=0.916)$. A linear regression analysis was carried out to evaluate accuracy of the method. The average $95 \%$ confidence interval of the best fit regression line $l=m y+c$ was $[-0.014,0.014]$ body weights, where $l$ is the predicted active force and $y$ is the measured active force. The calculated gradient and intercept values with corresponding $95 \%$ confidence intervals were $m=0.858$ [0.823, 0.893] and $c=0.368$ [0.277, 0.459] body weights, respectively. The average network training time was 0.64 seconds with Intel processor (core i7 2.6 GHz).

\section{Discussion}

In this work, a new data-driven, non-linear approach for biomechanical analysis of running was presented. It seems plausi-
Table 2: Ground reaction peak force values of young, middle-aged, and old groups during running

\begin{tabular}{llll}
\hline & Young & Middle-aged & Old \\
\hline Average peak vertical (BW) & $3.19^{*}$ & $2.95^{*}$ & $2.66^{*}$ \\
Abnormal high peak vertical (BW) & 3.74 & 3.46 & 2.97 \\
Difference (BW) & $\mathbf{0 . 5 5}$ & $\mathbf{0 . 5 1}$ & $\mathbf{0 . 3 1}$ \\
\hline *based on results in [15] & & & \\
BW = body weights & & &
\end{tabular}

ble that the accuracy of the current method is sufficient for discriminating between normal and abnormal peak vertical GRF values of recreational runners. As an example, GRF peak values of young, middle-aged, and old athletes were measured and compared in [15]. By calculating the 95th percentiles of the values for each age category, we obtained estimates of the thresholds for abnormal peak forces from these data sets. Mean GRF values, abnormal GRF values, and differences between the abnormal and mean values for each age category are listed in Table 2 . Clearly the difference between abnormal and mean GRF is much larger than the average standard error of the estimate obtained in this study ( 0.107 body weights), indicating that the current method is capable of discriminating runners with exceptionally high GRFs from runners having the average peak GRF values. In the future, the accuracy of the method can be further improved by collecting more training data.

The method is currently limited to predict only a peak GRF, but the goal in the future is to predict several variables of interest which allows comprehensive technical analysis of runners. To reach this goal, the effect of different sophisticated kinematic features will be investigated and the suitability of more advanced algorithms, such as those based on deep learning, will be tested. Previous studies suggest that especially vertical GRF impact peak [16][17] and loading rate [17] may increase the risk of running related injuries.

Peak force prediction from kinematics in running has not previously been reported in the literature. Some previous studies have focused on lower limb load prediction in walking [3][4] and jumping [18]. In walking, good correlations were achieved in [3] (0.991) and in [4] (0.999), when vertical GRFs were predicted. In [18], joint torques based on the GRF parameters during jumping were predicted and good correlations were obtained (over 0.950). Differences in the correlation values obtained in the current study and in the previous studies might be explained by different movement tasks, experimental arrangements, and predicted parameters. The total number of participants was considerably lower in previous studies (10 - 48 adults). Additionally, tenfold cross validation was used only in [3]. Moreover, speed is usually faster and contact time is shorter during running, when compared to speed and contact time while walking. Figure 1 shows how the running speed ranged from 2 to 
$6.5 \mathrm{~m} / \mathrm{s}$ in the current study. Further, in running, the total numbers of marker trajectories and locations may be much higher when compared to numbers in walking and jumping.

In this work, contact times were estimated from the motion vectors of the foot markers. If exact contact times were available as input variables, the average Pearson's correlation coefficient and standard error of the estimates would have been 0.926 and 0.103 body weights, respectively. However, the estimated contact times correlated very well with the values measured from a force platform. The correlation coefficient between estimated and exact contact times was 0.912 .

The implication of the current study is that it is possible to predict active vertical peak GRF during running basis of pure kinematic data. This has several practical implications in the context of human locomotion. First, the model can be used to assess runner's total leg force production outside limited laboratory space. Second, a fast processing time allows prediction of peak GRF in real time. Above all, the proposed approach may not only be beneficial for detecting runners with a high risk of injury, but it may be useful to analyze multiple aspects of running technique. Thus, based on the model output, personalized exercise programs could be offered to runners to prevent injuries and improve efficiency of their running.

\section{Conclusion}

An MLP model to predict active peak of the GRF was developed in this study. Weight and selected kinematic running parameters were used as an input features to the network. The MLP performance was evaluated using the Bayesian regularization training function and ten-fold cross validation technique. The standard error of the estimate and the Pearson's correlation coefficient were used to determine the prediction accuracy. The average value of standard error of the estimate was 0.107 body weights and the average value of Pearson's correlation coefficient was 0.919 . These results indicate that computational data-based methods allow prediction of the peak leg force generation during running and consequently enable scaling of biomechanical analysis for more extensive utilization. The study shows that the MLP model can be used as a flexible alternative for force platforms to discriminate runners with abnormal peak vertical GRF values.

Acknowledgements This study was supported by the Finnish Funding Agency for Technology and Innovation (Tekes) and the Academy of Finland.

\section{References}

1. van Mechelen W (1992) Running injuries. a review of the epidemiological literature. Sports Medicine 14(5):320-335

2. Keller TS, Weisberger AM, Ray JL, Hasan SS, Shiavi RG, Spengler DM (1996) Relationship between vertical ground reaction force and speed during walking, slow jogging, and running. Clinical Biomechanics 11(5):253-259

3. Oh SE, Choi A, Mun JH (2013) Prediction of ground reaction forces during gait based on kinematics and a neural network model. Journal of Biomechanics 46(14):2372-2380

4. Choi A, Lee JM, Mun JH (2013) Ground reaction forces predicted by using artificial neural network during asymmetric movements. International Journal of Precision Engineering and Manufacturing 14(3):475483

5. Corazza S, Mündermann L, Chaudhari AM, Demattio T, Cobelli C, Andriacchi TP (2006) A markerless motion capture system to study musculoskeletal biomechanics: visual hull and simulated annealing approach. Annals of Biomedical Engineering 34(6):1019-1029

6. Fernández-Baena A, Susín A, Lligadas X (2012) Biomechanical validation of upper-body and lowerbody joint movements of kinect motion capture data for rehabilitation treatments. Fourth International Conference on Intelligent Networking and Collacorative Systems pp 656-661

7. Ren L, Jones RK, Howard D (2008) Whole body inverse dynamics over a complete gait cycle based only on measured kinematics. Journal of Biomechanics 41(12):2750-2759

8. Ardestani MM, Zhang X, Wang L, Lian Q, Liu Y, He J, Li D, Jin Z (2014) Human lower extremity joint moment prediction: A wavelet neural network approach. Expert Systems with Applications 41(9):4422-4433

9. Staudenmann D, Robadey J, Lorenzetti S, Taube W (2015) Estimation of force, stiffness and elastic energy based on kinematic data while running. The 25 th Congress of the International Society of Biomechanics, Glasgow, UK, 2015

10. Bishop CM (2006) Pattern Recognition and Machine Learning. Springer-Verlag

11. Minsky M, Seymour P (1969) Perceptrons. MIT Press, Oxford

12. Duda RO, Hart PE, Stork DG (2001) Pattern Classification, 2nd Edition. Wiley-Interscience

13. Hornik K, Stinchcombe M, White H (1989) Multilayer feedforward networks are universal approximators. Neural Networks 2(5):359-366 
14. Foresee FD, Hagan MT (1997) Gauss-newton approximation to bayesian learning. International Joint Conference on Neural Networks 3:1930-1935

15. Kulmala JP, Korhonen MT, Kuitunen S, Suominen H, Heinonen A, Mikkola A, Avela J (2014) Which muscles compromise human locomotor performance with age? Journal of the Royal Society, Interface 11(100)

16. Zadpoor AA, Nikooyan AA (2011) The relationship between lower-extremity stress fractures and the ground reaction force: a systematic review. Clinical Biomechanics 26(1):23-28

17. Milner C, Ferber R, Pollard C, Hamill J, Davis I (2006) Biomechanical factors associated with tibial stress fracture in female runners. Medicine and Science in Sports and Exercise 38(2):323-328

18. Liu Y, Shih SM, Tian SL, Zhong YJ, Li L (2009) Lower extremity joint torque predicted by using artificial neural network during vertical jump. Journal of Biomechanics 42(7):906-911 